
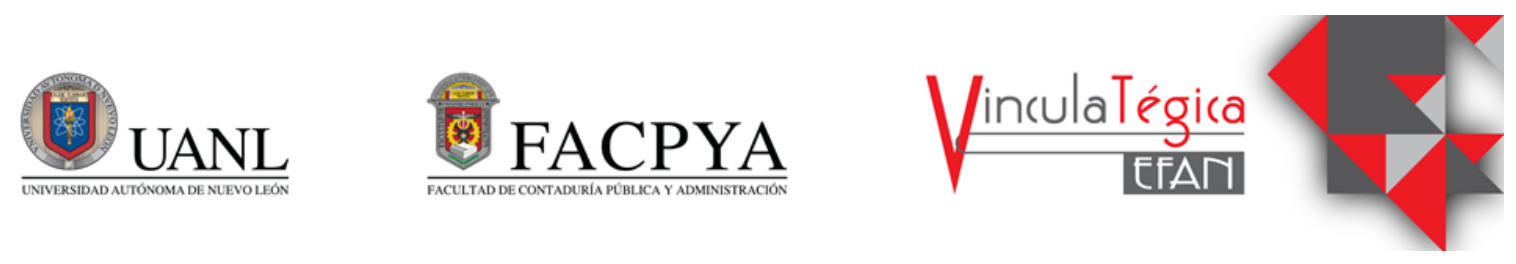

\title{
La concentración de los servicios de auditoría financiera en México. Caso aplicado a la Bolsa Mexicana de Valores
}

\author{
Daniel Alberto Cervantes Contreras ${ }^{1}$, Juan Rositas Martínez ${ }^{2}$ y Carlos Torres Gutiérrez ${ }^{3}$ \\ ${ }^{1}$ Universidad Autónoma de Nuevo León, Facultad de Contaduría Pública y Administración, Monterrey, Nuevo León, \\ mexicodaniel@hotmail.com, Av. Universidad S/N, Ciudad Universitaria, (+52) 8183204080. \\ ${ }^{2}$ Universidad Autónoma de Nuevo León, Facultad de Contaduría Pública y Administración, Monterrey, Nuevo León, \\ jrositasm@yahoo.com, Av. Universidad S/N, Ciudad Universitaria, (+52) 8183204080. \\ ${ }^{3}$ Universidad Autónoma de Nuevo León, Facultad de Contaduría Pública y Administración, Monterrey, Nuevo León, \\ cpcarlostorres@ hotmail.com, Av. Universidad S/N, Ciudad Universitaria, (+52) 8183204080.
}

Información del artículo revisado por pares

Fecha de aceptación: junio-2021

Fecha de publicación en línea: diciembre-2021

DOI: https://doi.org/10.29105/vtga7.2-28

\section{Resumen}

El estudio del grado de Concentración de los Servicios de Auditoría se ha ido desarrollando frecuentemente en diversos países, sin embargo, el análisis de este importante tema no se ha llevado a cabo en México en recientes años. El objetivo de este artículo de investigación es el presentar los resultados del estudio cuantitativo respecto a la Concentración de los Servicios de Auditoría en dicho país, basándonos en la información empresarial de carácter bursátil por los años 2018 a 2020, utilizando como método la aplicación del "Índice de Concentración" y el "Índice de Herfindahl". Los resultados indican que la Concentración de los Servicios de Auditoría por parte de las denominadas "Big 4", si bien sigue siendo alta, ha disminuido a través de los años.

Palabras clave: Concentración de los Servicios de Auditoría; Mercado de Auditorías; Empresas que cotizan en Bolsa; Firmas de Auditoría; índice de Herfindahl; Big 4; Big Four.

\section{INTRODUCCIÓN}

Desde la década de los 60's y 70's se dieron los indicios de las primeras investigaciones relacionadas al estudio de la composición del mercado en los servicios de auditoría; en México, carente ha sido la emisión de literatura sobre este tema tan particular en esta rama de la contaduría; las primeras publicaciones referentes a dicho ámbitto fueron 1498 1levvados a cabo en Estados Unidos de América (EU) hace cerca de 60 años, cuando

\begin{abstract}
The analysis of the concentration of audit services has been frequently developed in various countries, however, the study of this important issue has not been carried out in Mexico in recent years. The objective of this research article is to present the results of the empirical study regarding the Concentration of Audit Services in said country, based on business information of a stock market nature for the years 2018 to 2020, using the application of the "Concentration Indices" and the "Herfindahl Index". The results indicate that the concentration of Audit Services by the so-called "Big 4", although still high, has decreased over the years.
\end{abstract}

Keywords: Concentration of Audit Services; Audit Market; Listed Companies; Audit Firm; Herfindahl index; Big 4; Big Four.

Stephen A. Zeff y Robert L. Fossum (1967) publican "An Analysis of Large Audit Clients" (Análsis de Grandes Clientes de Auditoría) y John Grant Rhode, Gary M. Whitsell y Richard L. Kelsey (1974) publican "An Analysis of Client-Industry Concentrations for Large Public Accounting Firms" (Análisis de las Concentraciones de ClienteIndustria para Grandes firmas de Contabilidad

Pública). 
revelan la existencia de una alta participación de las "Big Eight", las ocho más grandes Firmas, en el mercado referente a los servicios de Auditoría. El concentrado de esas ocho más grandes firmas de Auditoría estaba conformado por: "Arthur Andersen \& Co.", "Arthur Young \& Company", "Ernst \& Ernst", "Haskins \& Sells", "Lybrand, Ross Bros. \& Montogmery", "Peat, Marwick, Mitchell \& Co.", "Price Waterhouse \& Co.", y "Touche, Ross, Bailey \& Smart" (Rhode et al., 1974).

En la actualidad, el término de las "Big Four" o "Big 4" es utilizado para hacer referencia a las firmas más importantes en este ámbito de la Consultoría y la Auditoría; este concepto está integrado por la presencia de las firmas "Deloitte Touche Tohmatsu Limited" (DELOITTE), "KPMG" (KPMG), "Ernst \& Young" (EY) y "PricewaterhouseCoopers" (PWC).

La importancia del presente artículo se cimienta en el hecho de que servirá de base para futuras investigaciones científicas que relacionen con el estudio de las Big Four, y se sustenta en el análisis de 409 auditorías, realizadas a varias empresas que cotizan en la Bolsa Mexicana de Valores (BMV), durante los años 2018, 2019 y 2020.

\section{Figura 1. Número de Auditorías a Empresas que cotizan en la BMV. \\ Número de Auditorías a Empresas que} cotizan en la BMV

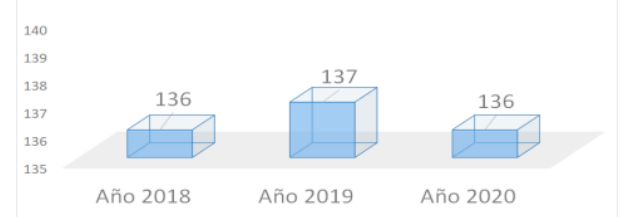

Fuente: Elaboración propia con información de la BMV.

\section{MARCO TEÓRICO}

\subsection{TEORÍA DE LA CONCENTRACIÓN EN LOS SERVICIOS DEL ÁREA DE AUDITORÍA}

El estudio del mercado de los servicios de auditoría, y su debida concentración, se apoya en el paradigma estructuralista y en el profundo de la teoría respectiva del poder de mercado (Toscano, 2010). El "paradigma estructuralista", se basa en el análisis de las relaciones que prevalecen entre la estructura, los comportamientos y los resultados, y un número basto de competidores para evitar una colusión al respecto, considerando que los resultados del mercado derivan de la función del tipo de competencia que existe dentro del mismo (competencia perfecta, competencia imperfecta, oligopolio, monopolio, y demás), ya que dicha competencia, de la misma manera, se encuentra muy relacionada con la estructuración del mercado y con su debido nivel de concentración económica. Respecto al "conocimiento profundo" del poder de mercado, aplicado al estudio de las ciencias contables, particularmente en la concentración de los servicios de auditoría, es pieza clave, ya que, en primera instancia, permite representar el factor estructural más importante del mercado de auditoría, $y$, en segunda instancia, ya que representa un indicador que engloba contenido informativo a gran escala, al producirse una relación entre el concepto de la concentración de los servicios de auditoría y entre el tipo de competencia que existe, esto significa que cuanto mayor sea el grado de concentración, más grande será el grado en que se aleja el mercado de la estructura teórica de lo que implica la competencia perfecta.

\subsection{LA CONCENTRACIÓN EN LOS SERVICIOS DEL ÁREA DE AUDITORÍA 2.2.1 LA CONCENTRACIÓN DE LOS SERVICIOS DE AUDITORÍA OTROS PAÍSES}

En la Tabla 1 se muestra una reseña de las investigaciones más relevantes que se han publicado durante las últimas décadas sobre el mercado y el grado de concentración en los servicios de la Auditoría en Estados Unidos.

Tabla 1. Estudios relevantes sobre el grado de concentración del mercado de auditoría en EU.

\begin{tabular}{|c|c|c|}
\hline $\begin{array}{l}\text { Autores y año de } \\
\text { publicación }\end{array}$ & $\begin{array}{c}\text { Metodologia, muestra u objetivo del trabajo } \\
\text { y años de estudio }\end{array}$ & Principales conclusiones o aportaciones \\
\hline Zeff y Fossum (1967) & $\begin{array}{c}\text { índices de concentración } \\
639 \text { empresas } 1964\end{array}$ & $\begin{array}{c}\text { Trabajo pionero } \\
\text { Alta concentración del } 94.8 \%\end{array}$ \\
\hline Rhode et all. (1974) & $\begin{array}{l}\text { Ińdices de concentración } \\
619 \text { empresas estadounidenses 1966-1971 }\end{array}$ & $\begin{array}{l}\text { Existe rivallidad entre grandes firmas, que } \\
\text { garantiza la calidad } \\
\text { Alta cancentración del } 96.45 \%\end{array}$ \\
\hline $\begin{array}{l}\text { Dopuch y Simunic } \\
\qquad(1980)\end{array}$ & $\begin{array}{l}\text { índices de concentración } \\
500 \text { empresas estadounidenses } 1977\end{array}$ & $\begin{array}{l}\text { Análisis de cuotas de mercado de las grandes } \\
\text { firmas por sectores Importancia de } \\
\text { especialización } \\
\text { Alta concentración del } 96.8 \%\end{array}$ \\
\hline $\begin{array}{c}\text { Eichenseher y Danas } \\
\text { (1981) }\end{array}$ & $\begin{array}{l}\text { Índice de Herfindahl } \\
8000 \text { registros } 1978\end{array}$ & $\begin{array}{l}\text { Introduce el Índice de Herfindahl como } \\
\text { medida de concentración Importancia del } \\
\text { tamaño } \\
\text { Índice de Herfindahl = }=0.15\end{array}$ \\
\hline $\begin{array}{c}\text { Danos y Eichenseher } \\
\text { (1982) }\end{array}$ & $\begin{array}{c}\text { Índice de Herfindahl } 33 \text { industrias } \\
1972-1979\end{array}$ & $\begin{array}{l}\text { Centrado en sectores económicos } \\
\text { Existe un efecto de reputación }\end{array}$ \\
\hline $\begin{array}{c}\text { Campbell y McNiel } \\
\text { (1985) }\end{array}$ & $\begin{array}{l}\text { Modelo estocástico } 650 \text { compañías } \\
\text { 1989/1994/1999/2004 }\end{array}$ & $\begin{array}{l}\text { Análisisis de cuotas por sectores } \\
\text { Importancia de especialización } \\
\text { Alta concentración: mâs del } 95 \% \\
\end{array}$ \\
\hline $\begin{array}{l}\text { Eichenseher y Danas } \\
\text { (1986) }\end{array}$ & Modelo Stigler & $\begin{array}{l}\text { Existen barreras de entrada } \\
\text { Existen economias de escala } \\
\text { y ventajas competitivas }\end{array}$ \\
\hline $\begin{array}{l}\text { Tonge y Wootton } \\
\text { (1991) }\end{array}$ & $\begin{array}{c}\text { Empresas que cotizan en las principales } \\
\text { Bolsas } \\
\text { Efecto de las fusiones }\end{array}$ & $\begin{array}{l}\text { Alta concentración: } 99 \% \text { Big } 6 \\
\text { Incremento potencial de la competencia }\end{array}$ \\
\hline $\begin{array}{l}\text { Minyardy Tabos } \\
(1991)\end{array}$ & $\begin{array}{l}\text { La concentración del mercado } \\
\text { antes y después de las fusiones }\end{array}$ & $\begin{array}{c}\text { Ningún efecto sobre competencia } \\
\text { entre grandes firmas auditoras }\end{array}$ \\
\hline Wootton et al. (1994) & Efecto de las fusiones 1991 & $\begin{array}{c}\text { No se incrementan las cuotas de mercado } \\
\text { después de la fusión }\end{array}$ \\
\hline Kwan (1996) & $\begin{array}{l}3820 \text { empresas cotizadas en la Bolsa de } \\
\text { Valores de Nueva York } 1989\end{array}$ & $\begin{array}{c}\text { Alta concentración: } 98 \% \text { Big } 6 \\
\text { Análisis con base en empresas auditadas }\end{array}$ \\
\hline $\begin{array}{c}\text { Doagar y Easley } \\
\text { (1998) }\end{array}$ & $\begin{array}{l}\text { Modelo de simulación } \\
\text { Muestra: } 5320 \text { compañías }\end{array}$ & $\begin{array}{c}\text { Alto nivel de concentración } \\
\text { por fusión proyectada }\end{array}$ \\
\hline Wolk et al. (2001) & $\begin{array}{c}\text { Efecto de las fusiones desde perspectiva } \\
\text { histórica } 1988 \text { a } 1999\end{array}$ & $\begin{array}{l}\text { Incremento significativo de concentración } \\
\text { del mercado para cuatro, seis y ocho grandes }\end{array}$ \\
\hline
\end{tabular}

Fuente: Tomado de "Análisis del mercado de servicios de auditoría en México" (Toscano, 2010).

En la "Tabla 2" se muestra un resumen de los estudios realizados en diversos países de Europa, sobre esta particular línea del área de la Auditoría. 
Tabla 2. Estudios relevantes sobre el grado de concentración del mercado de auditoría en Europa.

\begin{tabular}{|c|c|c|}
\hline $\begin{array}{l}\text { Autores y año de } \\
\text { publicación }\end{array}$ & $\begin{array}{c}\text { Metodología, muestra u objetivo del trabajo } \\
\text { y años de estudio }\end{array}$ & Principales conclusiones o aportaciones \\
\hline $\begin{array}{l}\text { Christiansen y Loft } \\
\text { (1992)/Dinamarca }\end{array}$ & $\begin{array}{c}\text { Efectos antes } \mathrm{y} \text { después de las fusiones } \\
1983,1989 \text { y } 1990\end{array}$ & $\begin{array}{l}\text { Se observa un incremento } \\
\text { en el nivel de concentración }\end{array}$ \\
\hline $\begin{array}{l}\text { Maijoor et al. } \\
\text { (1995)/Holanda }\end{array}$ & $\begin{array}{l}\text { Análisis y evolución de los índices } \\
1880 \text { a } 1990\end{array}$ & $\begin{array}{l}\text { Existe aumento constante } \\
\text { en concentración del mercado }\end{array}$ \\
\hline $\begin{array}{c}\text { Marten } \\
\text { (1996)/Alemania }\end{array}$ & $\begin{array}{c}\text { Utilización de subrogado en número } \\
\text { de clientes: } 250 \text { empresas grandes } 1900 \text { a } \\
1994 \\
\end{array}$ & $\begin{array}{l}\text { Alta concentración del mercado } \\
\text { En } 1990 \text { un } 59.6 \% \text { y } 65.8 \% \text { en } 1994\end{array}$ \\
\hline $\begin{array}{l}\text { Schaen y Maijoor } \\
(1997) / \text { Bélgica }\end{array}$ & $\begin{array}{l}\text { Análisis de estructura de mercado } \\
10500 \text { empresas auditadas }\end{array}$ & $\begin{array}{l}\text { Se observa estructura } \\
\text { del mercado dominante } \\
\text { Mantiene oligopolio flojo }\end{array}$ \\
\hline $\begin{array}{l}\text { Weets y Jegers } \\
\text { (1997)/Alemania }\end{array}$ & $\begin{array}{l}\text { Estudio comparativo } \\
1990 \text { y } 1993\end{array}$ & $\begin{array}{l}\text { Observan } 56 \% \text { del mercado } \\
\text { alemán para las Big } 6\end{array}$ \\
\hline $\begin{array}{l}\text { De Beelde } \\
\text { (1997)/Bélgica }\end{array}$ & $\begin{array}{l}11611 \text { grandes compañías } \\
\text { de } 14 \text { paises de Europa }\end{array}$ & $\begin{array}{l}\text { Nivel de concentración es variable } \\
\text { y el dominio de las Big } 6 \\
\text { en industrias, no es consistente }\end{array}$ \\
\hline $\begin{array}{c}\text { Quicky Wolz } \\
\text { (1999)/Alemania }\end{array}$ & $\begin{array}{c}\text { Estudio comparativo de concentración } \\
200 \text { empresas más grandes } \\
1991 \text { y } 1994\end{array}$ & $\begin{array}{l}\text { No se encuentran grandes } \\
\text { diferencias con respecto } \\
\text { a otros mercados }\end{array}$ \\
\hline $\begin{array}{l}\text { Buijink et al. } \\
\text { (1998)/Holanda }\end{array}$ & $\begin{array}{c}\text { Utilización de subrogado de personas } \\
\text { cualificadas que hacen auditorias } \\
1970 \text { a } 1994 \\
\end{array}$ & $\begin{array}{l}\text { Existe incremento en cuota } \\
\text { de mercado de grandes } \\
\text { firmas internacionales }\end{array}$ \\
\hline $\begin{array}{l}\text { García-Benau et al. } \\
\text { (1998)/Espan̂a }\end{array}$ & $\begin{array}{c}\text { Análisis de estructura del mercado } \\
\text { Empresas medianas y grandes } \\
1992 \text { a } 1994 \\
\end{array}$ & $\begin{array}{c}\text { Mercado español imperfecto } \\
\text { Competencia tipo oligopolio } \\
\text { Liderazgo de Andersen }\end{array}$ \\
\hline $\begin{array}{l}\text { García-Ayuso y } \\
\text { Sánchez } \\
\text { (1999)/España }\end{array}$ & $\begin{array}{l}1100 \text { informes publicados CNMV } \\
1991 \text { a } 1995\end{array}$ & El $41.9 \%$ los firma Andersen \\
\hline $\begin{array}{l}\text { Carrera et al. } \\
\text { (2005)/España }\end{array}$ & $\begin{array}{l}5510 \text { informes publicados CNMV } \\
1990-2000\end{array}$ & $\begin{array}{l}\text { Mercado español presenta } \\
\text { estructura oligopolística } \\
\text { Dominio de grandes firmas }\end{array}$ \\
\hline
\end{tabular}

Fuente: Tomado de "Análisis del mercado de servicios de auditoría en México” (Toscano, 2010).

GarcíaBenau et al. (1998), son considerados los precursores en estudiar el análisis de la estructura del mercado de los servicios de auditoría en España, toda vez que durante el periodo 1992-1994 utilizaron una muestra de empresas grandes de tamaño mediano y grande a las cuales les aplicaron el índice de concentración de orden " $n$ " y el índice de Herfindahl, llegando a observar que la concentración es debida a la inclinación de las empresas a ser auditadas por las firmas de carácter internacional. Como hallazgos principales destacan el hecho de que la firma Arthur Andersen, en el ejercicio 1994, atendía una cuota de mercado equivalente al 43\%, seguida por PWC que abarcaba el $12 \%$ del, mercado, a su vez que las firmas Coopers \& Lybrand y Ernst \& Young, abarcaban ambas un $10 \%$ del mercado, Peat Marwick se posicionaba en el lugar número cinco con el $9 \%$ de participación y en último lugar la firma Deloitte \& Touche con un 2\% del mercado; el análisis concluyó que el mercado de los servicios de auditoría en España es imperfecto y existiendo una competencia del tipo oligopolística. De la misma manera, García-Ayuso y Sánchez (1999) analizaron el grado de concentración por el periodo de 1991 a 1995, estudio a través del cual encontraron que el 41.9\% de las auditorías fueron realizadas por la firma Arthur Andersen, seguida por las firmas Ernst and Young y Peat Marwick quienes dictaminaron un $13 \%$ y un $13.09 \%$ respectivamente; seguido de estas firmas, figuró Price Waterhouse quien signó 133 representando un $10.9 \%$ del mercado, en tanto que Deloitte \& Touche y Coopers \& Lybrand auditaron un $3.63 \%$ y $5.36 \%$, respectivamente. Por su parte, Nieves et al (2005), en su investigación consistente en la estructura del mercado de auditoría en la nación Española, por el periodo 1990-2000, comprobaron que el mercado de auditoría muestra una estructura del tipo oligopólica, dominada por las grandes firmas de carácter internacional (Toscano, 2014).

En 2012, Heß y Stefani publican “Audit Market Regulation and Supplier Concentration Around the World: Empirical Evidence", investigación que deriva de la preocupación respecto a que las regulaciones destinadas a mejorar la independencia puedan incrementar aún más la concentración de la auditoría. A través de este estudio llegan a la conclusión de que los organismos reguladores deben tener en cuenta las conexiones que existen en el estudio de la auditoría: en primera instancia, la existencia de un sistema de responsabilidad y la prohibición del suministro conjunto de servicios de auditoría y relacionados reduce significativamente la concentración; así mismo, las auditorías conjuntas y la rotación obligatoria de las firmas de auditoría aumentan significativamente la concentración del mercado de auditoría. En este estudio exponen los indicadores de concentración de algunos países, determinados con información de 2001 a 2010, como se muestra en la Tabla 3.

Tabla 3. Índices de Herfindahl aplicado a los servicios

\begin{tabular}{|l|c|}
\multicolumn{2}{|c}{ de Auditoría. } \\
\hline Australia & $\begin{array}{c}\text { índicede } \\
\text { Herfindah }\end{array}$ \\
\hline Austria & 0.077 \\
\hline Bélgica & 0.188 \\
\hline Brasil & 0.119 \\
\hline Canadá & 0.134 \\
\hline República Checa & 0.128 \\
\hline Dinamarca & 0.216 \\
\hline Estonia & 0.373 \\
\hline Finlandia & 0.278 \\
\hline Francia & 0.097 \\
\hline Alemania & 0.084 \\
\hline Grecia & 0.219 \\
\hline Hungria & 0.174 \\
\hline Irlanda & 0.257 \\
\hline Italia & 0.209 \\
\hline Japón & 0.171 \\
\hline Corea & 0.107 \\
\hline Luxemburgo & 0.168 \\
\hline Países Bajos & 0.205 \\
\hline Noruega & 0.264 \\
\hline Polonia & 0.064 \\
\hline Portugal & 0.161 \\
\hline Eslovenia & 0.330 \\
\hline España & 0.257 \\
\hline Suecia & 0.204 \\
\hline Suiza & 0.262 \\
\hline Turquía & 0.115 \\
\hline Estados Unidos & 0.105 \\
\hline Países de la Unión Europea & 0.106 \\
\hline
\end{tabular}

Fuente: Tomado de Heß y Stefani (2012) "Audit Market Regulation and Supplier Concentration Around the World: Empirical Evidence".

Como se muestra en la tabla que antecede, los países que presentan el mayor indicador de concentración en los servicios de Auditoría, de conformidad con el índice de Herfindahl, son Dinamarca y Portugal con 
resultados de 0.373 y 0.330 , respectivamente; por el contrario, entre los países que muestran menor concentración están Australia, Francia y Noruega, con indicadores de $0.077,0.084$ y 0.064 , respectivamente.

Respecto a América Latina, como ya se expuso en el estudio de Heß y Stefani (2012), Brasil obtuvo un indicador de 0.0134, lo que evidencia que, en promedio, entre 7 y 8 es el número de firmas auditorías que concentran mayormente la revisión a las empresas que cotizan en la bolsa de valores de este país.

En Argentina, en el año 2017 la Firma de Auditoría "BDO", emitió un estudio que trata respecto a la concentración de los servicios de auditoría por los Ejercicios 2015 y 2016, en los que destacan el hecho de que la firma PWC encabeza la lista de las firmas auditoras, cifras que se muestran en la "Tabla 4".

Tabla 4. Número de Empresas auditadas por Firma de Auditoría en Argentina durante 2015-2016

\begin{tabular}{|c|c|c|}
\hline Firma Auditora & 2015 & 2016 \\
\hline PWC & 294 & 300 \\
\hline EY & 205 & 222 \\
\hline Delotte & 221 & 186 \\
\hline KPMG & 80 & 102 \\
\hline BDO & 45 & 50 \\
\hline Lisicki Litvin & 23 & 39 \\
\hline Auren & 17 & 32 \\
\hline RSM & 23 & 27 \\
\hline Grant Thornton & 23 & 22 \\
\hline Mazars & - & 16 \\
\hline Crowe Horwath & 10 & 13 \\
\hline Bertora & 9 & 8 \\
\hline Total & 950 & 1,017 \\
\hline
\end{tabular}

Fuente: Tomado de BDO Argentina (2017) "Las Mayores Compañías de Auditoría en Argentina".

De conformidad con un estudio realizado por la Firma PWC (2018), en Chile, todos los años, las "Big 4" se disputan la auditoría de la información financieros de las 40 empresas del Índice de Precios Selectivo de Acciones (IPSA). La firma EY sumó a 12 empresas auditadas del IPSA, concentrando el $30 \%$ del total de las 40 empresas, mientras que PWC atendió la misma cantidad. En el tercer puesto se posicionó DELOITTE, con 9 compañías que representan el $22.5 \%$ del total, y la firma KPMG fue contratada por 6 compañías, es decir, el 15\%, y la firma BDO sumó auditó una de estas empresas, que representa el $2.5 \%$ del mercado.

En un estudio de Gutiérrez et al. (2019), en el que se estudia la Concentración de los servicios de Auditoría en las empresas que cotizan en la bolsa de valores de Colombia, se evidencia que en los últimos años analizados (2017-2018), el Índice de Concentración nivel 4 ( $\left.\mathrm{IC}_{4}\right)$, que corresponde a la participación de las "Big 4", oscila entre el 96 y el $99 \%$.

\subsubsection{LA CONCENTRACIÓN DE LOS SERVICIOS DE AUDITORÍA EN MÉXICO} 2.2.2.1 Breve historia y regulación

El comienzo del mercado en los servicios de Auditoría, en los Estados Unidos Mexicanos, data desde inicios del siglo XX con el surgimiento de la carrera profesional de la Contaduría Pública y con la llegada de las grandes firmas de carácter multinacional. En la época de los años 30's se constituyen las iniciales firmas mexicanas de Auditoría que se irían fusionando con las firmas multinacionales a partir de los años 50's (Toscano, 2010).

La "Ley del Mercado de Valores" (LMV) es el ordenamiento que obliga a las empresas que cotizan en la BMV a dictaminarse, ya que en el artículo 43 establece que "los presidentes de los comités que lleven a cabo las funciones en materia de prácticas societarias y de auditoría deben elaborar un informe de carácter anual sobre las actividades que correspondan a estos órganos, el cual debe contemplar, entre otros aspectos, un dictamen de auditoría externa"; en el artículo 87 de la mencionada ley se establece la normativa a la que debe ajustarse la elaboración de dicho dictamen, entre otras indicaciones señala:

"I. El dictamen de auditoría externa deberá ser elaborado con base en normas y procedimientos de auditoría emitidas o reconocidas por la Comisión y, en todo caso, deberá versar sobre:

a) La razonabilidad de la información financiera.

b) El apego a los principios de contabilidad aplicables.

c) Los estados financieros elaborados por la emisora". (LMV, 2021)

\subsubsection{Estudios empíricos en México}

Después de una búsqueda sobre documentos relacionados con el tema de la Concentración en los Servicios del área de Auditoría, encontramos que antes de 2010 no se había realizado estudio alguno sobre este tema en México. No es sino hasta dicho 2010 cuando se surge la investigación "Análisis del mercado de servicios de auditoría en México", llevado a cabo por Toscano (2010) en el cual analiza la composición del mercado de los servicios del área de auditoría durante los años de 2001 a 2005; no encontramos investigaciones adicionales, después del citado estudio, sobre este tema en México, que analice lo sucedido en dicho mercado durante los 15 años posteriores a 2005. Motivo de lo anterior es que vemos necesario realizar un estudio que evidencie qué tanto ha variado la multicitada concentración en 
los servicios de esta rama de la Contaduría desde aquellas épocas, hasta la actualidad.

\subsubsection{Sobre la Auditoría Financiera para Efectos Fiscales}

Como comenta Hernández et al (2018), hasta el año 2013, atendiendo al Artículo 32-A del Código

Fiscal de la Federación y el Anexo 5 de la primera Resolución Miscelánea Fiscal, las personas morales residentes en México, de la misma manera que las Personas Físicas que llevaban a cabo una Actividad Empresarial, que hubieran tenido ingresos acumulables superiores a los ciento nueve millones de pesos, o que el valor invertido en sus activos haya sido superior a los ochenta y siete millones de pesos, o que hubieran recibido el servicio de al menos trescientos trabajadores, estaban obligados a que se les llevar a cabo una auditoría financiera para efectos fiscales, también denominada como "Dictamen Fiscal". Además de lo anterior, los contribuyentes que desearan podían optar por dictaminar sus estados financieros para efectos fiscales, sin embargo a partir del Ejercicio Fiscal 2014 despareció la opción comentada, permitiendo dictaminarse para efectos fiscales solamente los contribuyentes que cumplieran con los requisitos citados respecto al nivel de sus ingresos, al valor del activo o a la cantidad de personal empleado.

La obligatoriedad citada en el párrafo que antecede, se eliminó en atención a los puntos que destacan Yáñez \& Ávila (2015): en parte inicial debido a que el Poder Ejecutivo reconoce que el sistema tributario es complejo y oneroso, seguido del hecho de que el dictamen fiscal es un instrumento de carácter fiscalizador y representa un sustancial costo para el contribuyente, el cual es adicional al costo normal que implica los propios registros contables y el coste que implica el cumplir con las obligaciones fiscales.

Siguiendo con Hernández et al (2018), el hecho de volver optativo al Dictamen Fiscal, ha puesto en duda el beneficio de llevarlo a cabo, atendiendo principalmente al costo que conlleva el realiza esta práctica de auditoría.

Lo anterior ha implicado que firmas de auditoría de tamaño mediano hayan salido del mercado de la prestación de los servicios de auditoría en la nación mexicana.

En este apartado se presentaron algunos de los estudios presentados por otros investigadores en México y en distintas naciones respecto al estudio de la concentración de los servicios de Auditoría, por lo que a continuación se procederá a la aplicación de la metodología correspondiente para realizar el análisis objeto del presente estudio.

\section{MÉTODO}

3.1 INDICADORES DE CONCENTRACIÓN EN LOS SERVICIOS DEL ÁREA DE AUDITORÍA

Señalan García \& Novejarque (2009), que las investigaciones llevados a cabo en los distintos países emplean una metodología similar, la cual se basa en el citado "paradigma estructuralista", y derraman similares resultados a través de los diversos índices de concentración del mercado de los servicios de auditoría. De la misma manera, Hernández (2010) hace referencia a que en México la concentración de los servicios de auditoría, arroja prácticamente equiparables a los resultados de las investigaciones realizadas a nivel mundial.

En los puntos siguientes expondremos las técnicas que se emplea respecto a la determinación de los citados indicadores de concentración, para llevar a cabo el análisis de los datos obtenidos y evidenciar la evolución que ha tenido la concentración de los servicios de auditoría en México.

\subsubsection{Descripción del Índice de Concentración}

Como lo señalan Nieves et al. (2005) la concentración puede ser medida en la proporción de las cuotas de participación en el mercado de todas las compañías o algunas de ellas; sin embargo, la medida de medición más simple es el denominado "Índice de Concentración" de " $n$ " empresas $\left(\mathrm{IC}_{\mathrm{n}}\right)$ que viene a representar la cuota de las " $n$ " empresas que tienen más actividad en determinada industria. Interpretación: lo anterior indica, por ejemplo, que un índice de $\mathrm{IC}_{4}$ del 0.50 revela que las 4 empresas con más operación en el mercado poseen la mitad de la participación en él. El número elegido de empresas puede ser designado de manera arbitraria, dependiendo del objetivo del cálculo y del mercado que se esté estudiando.

El índice de concentración es calculado de la siguiente manera:

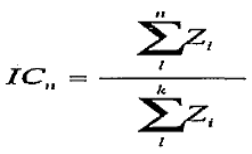

En donde:

$\mathrm{Z}_{\mathrm{i}}$ : es el "tamaño de la firma auditora", el cual se mide por el número de clientes.

$n$ : es el "número de empresas más grandes" sobre el que se calcula el índice.

$k$ : es el "número total de empresas auditoras" las cuales están activas en el mercado.

\subsubsection{Descripción del Índice de Herfindahl}


Siguiendo con Nieves et al. (2005) otra de las medidas que pueden utilizarse para medir la concentración, tomando como base las cuotas del marcado, es el "índice de Herfindahl" (IH). Este indicador permite tener considerar, por un lado, el número de empresas que permanecen activas $\mathrm{y}$, por otro lado, la variación en los niveles de actividad de dichas empresas. Interpretación: si el valor de este índice es equivalente a 1 , se estaría evidenciando que solo hay activa una empresa en el mercado, mientras que si este indicador tiende a valor 0 , estaríamos en presencia de un mercado donde participa una gran cantidad de empresas de similar tamaño. Este índice se calcula de la siguiente manera:

$$
I H I=\frac{\sum_{l}^{k}\left(Z_{i}\right)^{2}}{\left(\sum_{t}^{k} z_{i}\right)^{2}}
$$

En donde:

$\mathrm{Z}_{\mathrm{i}}$ : es el "tamaño de la firma auditora", que se mide por el número de clientes.

$k$ : es el "número total de empresas auditoras" que están activas en el mercado.

Diversos autores indican que es poco probable que la elección entre diversos indicadores de concentración pudiera afectar a las inferencias obtenidas respecto a la concentración en el mercado de los servicios de auditoría. No obstante lo anterior, otros autores acogen la utilización del "índice de Herfindahl" debido a que toma en consideración todas las auditorías activas, otorgando una idea que se aproxima más al nivel real de concentración.

\subsection{POBLACIÓN DE ESTUDIO}

En la presente investigación cuantitativa, cuyo objeto es la determinación de la Concentración de los Servicios del área de Auditoría en México, delimitamos el análisis al segmento de las auditorías efectuadas a las empresas que cotizan en la Bolsa Mexicana de Valores, durante los años 2018, 2019 y 2020, conforme se puede percibir en la "Tabla 5".

Tabla 5. Descripción de la Muestra a Estudiar.
\begin{tabular}{|c|c|c|c|}
\hline Concepto & 2018 & 2019 & 2020 \\
\hline $\begin{array}{c}\text { Número de dictámenes emitidos } \\
\text { a empresas auditadas }\end{array}$ & 136 & 137 & 136 \\
\hline $\begin{array}{c}\text { Número de firmas auditoras } \\
\text { que han ofrecido el servicio }\end{array}$ & 14 & 15 & 14 \\
\hline
\end{tabular}

Fuente: Elaboración propia con información de la BMV.

En este punto es importante comentar que, para efectos de llevar a cabo la metodología de la presente investigación, no se contempla a las
Empresas que actualmente pudieran estar enlistadas en la BMV, pero que durante los años de estudio no fueron auditadas: ya sea porque la acción de la empresa fue suspendida, por falta de liquidez, y demás causas.

Para determinar la composición del mercado de Auditoría, utilizaremos los ya definidos "Índice de Concentración" e "Índice de Herfindahl", mismos que ya han sido confirmados en los estudios de las áreas de la economía.

\section{RESULTADOS 4.1 RESULTADOS OBTENIDOS PARA MÉXICO}

\subsubsection{Resultados - Cuotas de Mercado}

Los resultados provenientes del empleo del método de medición de cuota de mercado, para las auditorías por los Ejercicios 2018, 2019 y 2020, se muestran en la "Tabla 6", en la que puede apreciarse que solo una firma de auditoría abarca casi la mitad del mercado de las auditorías para las grandes empresas.

Tabla 6. Cuotas de participación en el mercado por parte de las Firmas de Auditoría

\begin{tabular}{|c|c|c|c|}
\hline Firma de Auditoría & $\mathbf{2 0 1 8}$ & $\mathbf{2 0 1 9}$ & $\mathbf{2 0 2 0}$ \\
\hline DELOITTE & 0.40 & 0.41 & 0.41 \\
\hline KPMG & 0.20 & 0.19 & 0.18 \\
\hline PWC & 0.14 & 0.14 & 0.12 \\
\hline EY & 0.14 & 0.14 & 0.16 \\
\hline OTRAS & 0.12 & 0.12 & 0.13 \\
\hline TOTAL & $\mathbf{1 . 0 0}$ & $\mathbf{1 . 0 0}$ & $\mathbf{1 . 0 0}$ \\
\hline
\end{tabular}

Fuente: Elaboración propia con información de la BMV.

Figura 2. Cuotas de participación en el mercado por parte de las Firmas de Auditoría.

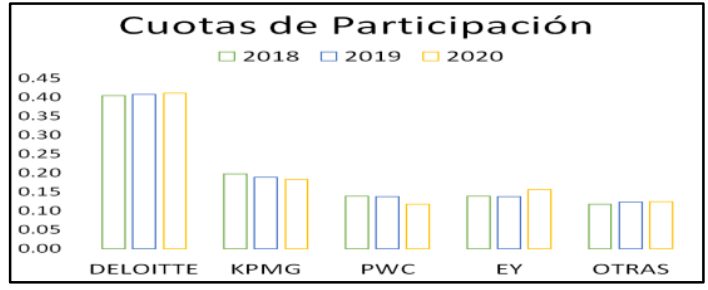

Fuente: Elaboración propia con información de la BMV.

\subsubsection{Resultados-Índices de Concentración}

Para este estudio fue importante determinar el $\mathrm{IC}_{1}$, con el objetivo de conocer la concentración de la firma de auditoría que posee la cuota más grande de participación en el mercado; así mismo también importante el determinar el $\mathrm{IC}_{2}$ y el $\mathrm{IC}_{4}$, con el objetivo de conocer el grado de concentración que poseen, respectivamente, las dos y las cuatro firmas de auditoría de más peso en los servicios de auditoría en México.

Dado lo anterior, los resultados obtenidos fueron los siguientes: 
Tabla 7. Resultados de los "Índices de Concentración”.

\begin{tabular}{|c|c|c|c|}
\hline $\begin{array}{c}\text { findice de } \\
\text { Concentración }\end{array}$ & 2018 & 2019 & 2020 \\
\hline $\mathrm{IC}_{1}$ & 0.40 & 0.41 & 0.41 \\
\hline $\mathrm{IC}_{2}$ & 0.60 & 0.60 & 0.59 \\
\hline $\mathrm{IC}_{3}$ & 0.74 & 0.74 & 0.70 \\
\hline $\mathrm{IC}_{4}$ & 0.88 & 0.88 & 0.87 \\
\hline
\end{tabular}

Fuente: Elaboración propia con información de la BMV.

Como se expuso en el apartado teórico, un índice de $\mathrm{IC}_{4}$ del 0.50 , por ejemplo, revelaría que las 4 compañías con el más alto grado de operación en el mercado perciben la mitad de la participación en él. Para el caso particular de esta investigación, el $\mathrm{IC}_{1}$ revela que para el 2018 la firma que obtuvo la mayor participación en el mercado tuvo un índice de concentración del 40\%, para el 2019 el $41 \%$ y para el 2020 el 41\%. El resultante $\mathrm{IC}_{2}$ nos indica que las 2 firmas que tuvieron mayor participación en el mercado, tuvieron un índice de concentración del $60 \%, 60 \%$ y $59 \%$, para 2018, 2019 y 2020, respectivamente. $\mathrm{Y}$ el $\mathrm{IC}_{4}$, nos muestra que las 4 firmas que tuvieron mayor participación concentraron el $88 \%, 88 \%$ y $87 \%$ respectivamente para los ejercicios 2018, 2019 y 2020.

\subsubsection{Resultados - Índices de Herfindahl}

El producto del empleo del método "Índice de Herfindahl", para las auditorías por los Ejercicios 2018, 2019 y 2020, se muestra en la "Tabla 8", que a continuación se expone:

Tabla 8. Resultados de los Índices de Herfindahl para los servicios de Auditoría.

\begin{tabular}{|c|c|c|c|}
\hline Índice de Herfindahl & 2018 & 2019 & 2020 \\
\hline $\begin{array}{c}\text { Por número de } \\
\text { empresas auditadas }\end{array}$ & 0.24 & 0.24 & 0.25 \\
\hline
\end{tabular}

Fuente: Elaboración propia con información de la BMV.

Como se explicó en el apartado "Marco Teórico", si el valor de este índice de Herfindahl es equivalente a 1 , se estaría evidenciando que solo hay activa una organización participante en el mercado, en tanto que si este indicador tiende a valor 0 , estaríamos en presencia de un mercado donde participa una gran cantidad de empresas de similar tamaño. Dados los resultados expresados en la "Tabla 8", se podría interpretar que el mercado de los servicios del área de auditoría, en el segmento investigado, prácticamente está concentrado en 4 firmas de auditoría para los años 2018, 2019 y 2020 , lo cual resulta de dividir la unidad entre el índice de Herfindahl para cada ejercicio.

\subsubsection{Resultados-Sector de especialización}

A manera de agregado, y como parte del resultado obtenido, en las Tablas 9 y 10 exponemos los sectores a los cuales estas grandes Firmas dan servicio, para tener una noción respecto a las esferas económicas en las cuales pudieran estar especializados.

Tabla 9. Sector de especialización por Firma de Auditoría integrante de las “Big 4", durante 2020.

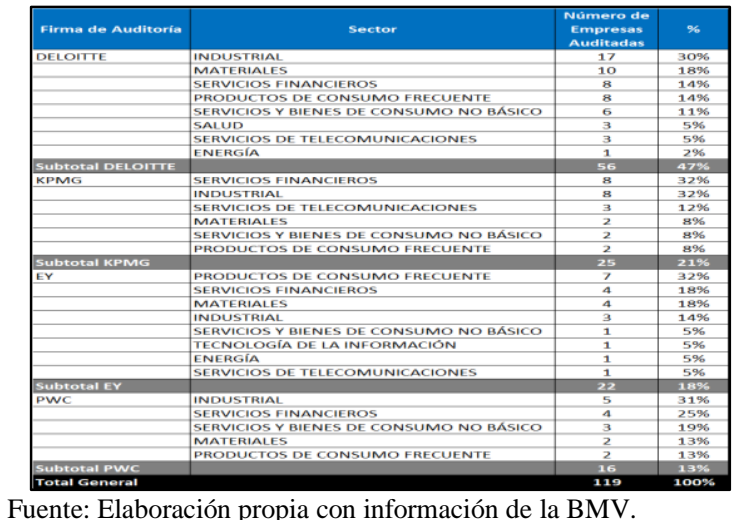

Tabla 10. Sector de atención por tipo de Firma de Auditoría, durante 2020.

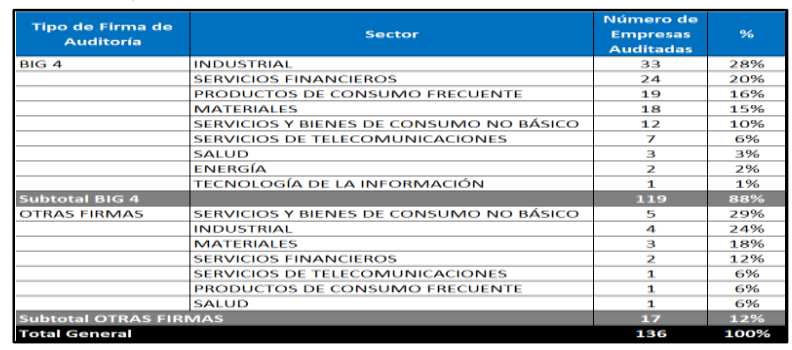

Fuente: Elaboración propia con información de la BMV.

\subsection{DE LA METODOLOGÍA APLICADA A ESTUDIOS REALIZADOS A OTROS PAÍSES}

Tomando como referencia el estudio realizado por Heß y Stefani (2012), en el que se exponen los índices de Herfindahl para los distintos países expuestos, procedemos a realizar el cálculo del número de Firmas que concentran los servicios de auditoría en las empresas que cotizan en la bolsa de valores de cada país, con lo que llegamos a la obtención del número de Firmas que concentran mayormente los servicios de Auditoría, mismos que se exponen en la "Tabla 11".

Tabla 11. Número de Firmas Auditoras que concentran los servicios de Auditoría en diversos países 


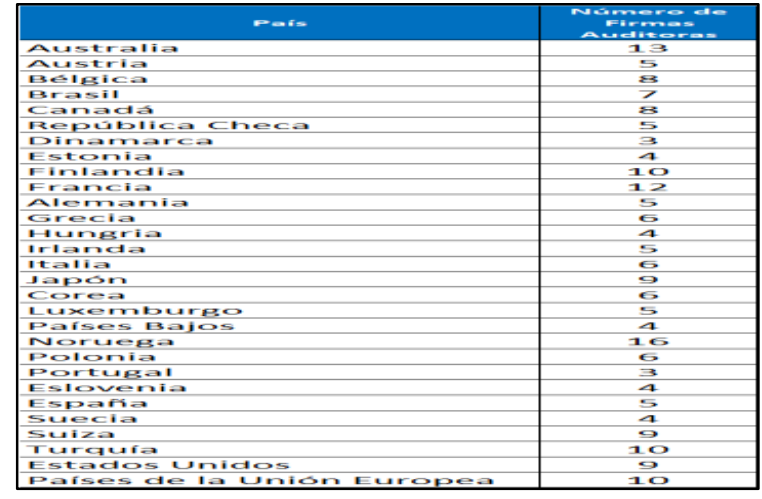

Fuente: Elaboración propia con información de Heß y Stefani

(2012).

Como se muestra en la tabla que antecede, con base el índice de Herfindahl, entre los países que poseen el menor número de Firmas de Auditoría que concentran estos servicios, son Dinamarca y Portugal con un resultado de en promedio 3 Firmas de Auditoría; por el contrario, entre los países que poseen el mayor número de Firmas que concentran los servicios de Auditoría, están Australia, Francia y Noruega, con 13, 12 y 16 Firmas en promedio, respectivamente. De manera conjunta, en los países de la Unión Europea la concentración de los servicios de auditoría en las empresas que cotizan en la bolsa de valores está concentrada en promedio en 10 firmas Auditoras.

Respecto al estudio hecho por BDO (2017), en el que se muestran los números de Firmas de Auditoría que atendieron el mercado Argentino, y aplicando el índice de Concentración "IC" a distintos niveles, logramos mostrar el grado en que las "Big 4" atienden este mercado, información que se revela en la "Tabla 12".

Tabla 12. Índice de Concentración de los Servicios de Auditoría en Argentina

\begin{tabular}{|c|c|c|}
\hline Índice de Concentración & $\mathbf{2 0 1 5}$ & $\mathbf{2 0 1 6}$ \\
\hline $\mathrm{IC}_{1}$ & 0.31 & 0.29 \\
\hline $\mathrm{IC}_{2}$ & 0.54 & 0.51 \\
\hline $\mathrm{IC}_{3}$ & 0.76 & 0.70 \\
\hline $\mathrm{IC}_{4}$ & 0.84 & 0.80 \\
\hline
\end{tabular}

Fuente: Elaboración propia con información de BDO (2017).

Como se logra ver en la tabla que antecede en la nación Argentina, para 2015 y 2016, los índices de Concentración de las "Big 4" redundan entre el 84 y $86 \%$ respectivamente para cada año.

Respecto a la nación de Chile, aplicando el índice de Concentración "IC" podemos obtener el grado de Concentración que poseen las grandes firmas, como se muestra en la "Tabla 13".

\section{Tabla 13. Indice de Concentración de los Servicios de} Auditoría en Chile

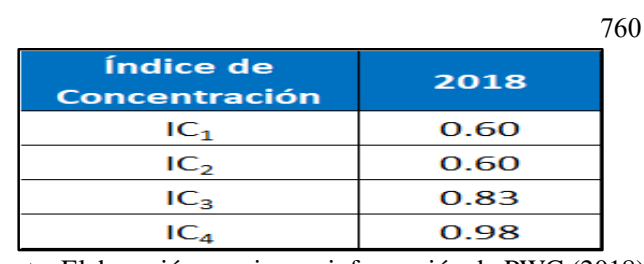

Fuente: Elaboración propia con información de PWC (2018).

Se puede apreciar en la tabla que antecede que índice de Concentración nivel 4, que corresponden a las "Big 4", en los servicios de Auditoría en la nación chilena, aplicado a los dictámenes realizados a empresas que forman parte del IPSA, corresponde al $98 \%$.

\subsection{ANÁLISIS COMPARATIVO}

A continuación expondremos breves comparativos de la información obtenida a través del resultado de nuestra investigación, contra los hallazgos obtenidos por otros investigadores en sus estudios empíricos.

\subsubsection{Comparativo contra años anteriores}

Siguiendo con la investigación de Toscano (2010), y con los resultados que se lograron obtener mediante del desarrollo del presente trabajo, es posible evidenciar distintos puntos, a saber:

1) De los años 2003 a 2005 la Firma de Auditoría que prestaba servicio a la mayor parte del mercado (EY) poseía entre el 26 y el $32 \%$ de participación respecto al total, de conformidad con el "Índice de Concentración". Para años recientes (2018 a 2020) la Firma que encuadra en este indicador (DELOITTE) posee entre el $40 \mathrm{y}$ $41 \%$ de participación. (Ver "Tabla 6" y “Tabla 13").

Tabla 13. Índice de Concentración nivel $1\left(I_{1}\right) 15$ años después.

Índice de Concentración nivel $1\left(\mathrm{IC}_{1}\right)$ Año 1 Año 2 Año 3 \begin{tabular}{|l|l|l|l|l|}
\hline $\mathrm{IC}_{1}$ (Año1/2003, Año2/2004, Año3/2005) & 0.26 & 0.31 & 0.32 \\
\hline
\end{tabular}

\begin{tabular}{|l|l|l|l|l|}
\hline $\mathrm{IC}_{1}$ (Año1/2018, Año2/2019, Año3/2020) & 0.40 & 0.41 & 0.41 \\
\hline
\end{tabular}

Fuente: Elaboración propia con información de Toscano (2010) e información resultante de la presente investigación.

2) Si bien, como se comentó en el inciso anterior, una Firma de Auditoría incrementó su nivel de concentración, de manera general, la participación de las "Big 4" en el mercado total se ha visto disminuida a través de los años, ya que de 2003 a 2005 su participación estaba entre el 94 y $96 \%$ y en años recientes (2018 a 2020) la participación conjunta de dichas firmas abarca entre el 87 y el $88 \%$, de conformidad con los cálculos del Índice de Concentración nivel $4\left(\mathrm{IC}_{4}\right)$ y las Cuotas de participación en el mercado, como se muestra en las Tablas 14 y 15, 
respectivamente.

Tabla 14. Índice de Concentración nivel 4 (IC 4$) 15$ años después.

índice de Concentración nivel $4\left(\mathrm{IC}_{4}\right)$ Año 1 Año 2 Año 3 \begin{tabular}{|l|l|l|l|}
\hline $\mathrm{IC}_{4}$ (Año1/2003, Año2/2004, Año3/2005) & 0.96 & 0.94 & 0.95 \\
\hline
\end{tabular} \begin{tabular}{|l|l|l|l|}
\hline $\mathrm{IC}_{4}$ (Año1/2018, Año2/2019, Año3/2020) & 0.88 & 0.88 & 0.87 \\
\hline
\end{tabular}

Fuente: Elaboración propia con información de Toscano (2010) e información resultante de la presente investigación.

Tabla 15. Cuota de participación en el mercado de las "Big 4" 15 años después.

\begin{tabular}{|c|c|c|c|}
\hline Cuota de Concentración & Año 1 & Año 2 & Año 3 \\
\hline BIG4 (Año1/2003, Año2/2004, Año3/2005) & 0.96 & 0.94 & 0.95 \\
\hline BIG4 (Año1/2018, Año2/2019, Año3/2020) & 0.88 & 0.88 & 0.87 \\
\hline Decremento & $\mathbf{0 . 0 8}$ & $\mathbf{0 . 0 6}$ & $\mathbf{0 . 0 8}$ \\
\hline
\end{tabular}

Fuente: Elaboración propia con información de Toscano (2010) e información resultante de la presente investigación.

3) El "Índice de Herfindahl" para los años 2003 a 2005 oscilaba entre el 0.17 y 0.19 , lo que indicaba que el mercado correspondiente a los servicios del área de Auditoría, en el segmento investigado, prácticamente estaba concentrado en 6 firmas de Auditoría; sin embargo, para años recientes, este indicador evidencia que dicho mercado prácticamente está concentrado en 4 Firmas Auditoras.

Tabla 16. Índice de Herfindahl 15 años después.

\begin{tabular}{|c|c|c|c|}
\hline Índice de Herfindahl & Año 1 & Año 2 & Año 3 \\
\hline $\mathrm{IH}$ (Año1/2003, Año2/2004, Año3/2005) & 0.19 & 0.17 & 0.17 \\
\hline $\mathrm{IH}$ (Año1/2018, Año2/2019, Año3/2020) & 0.24 & 0.24 & 0.25 \\
\hline
\end{tabular}

Fuente: Elaboración propia con información de Toscano (2010) e información resultante de la presente investigación.

\subsubsection{Comparativo contra otros países}

Con base en las investigaciones previas analizadas en el presente estudio, podemos realizar la comparativa constatando que para finales del año 2000 en España, las "Big 4" poseían participación de entre el 76 y $77 \%$ (Nieves et al., 2005), mientras que en México dicha participación, oscilaba entre el 94 y 96\%. Sin embargo, para 2009, en España, estas 4 grandes firmas concentraban el $98.42 \%$ del mercado de auditoría en las empresas cotizantes de la bolsa (Cuesta \& Polo, 2015), cifra que se asemeja a los índices de concentración que poseía la nación mexicana en aquella década.

Respecto a algunos países de América Latina, podemos mencionar a Chile, que, tal y como ya se expuso en los apartados anteriores de este trabajo, cuenta con un indicador del 98\% (2018) de concentración del mercado de los servicios de auditoría en las empresas que cotizan en la bolsa de valores por parte de las "Big 4", porcentaje que es mayor en gran medida respecto al índice de concentración correspondiente a México (88\%).

La nación Brasileña cuenta con un índice de Herfindahl de 0.0134 , que indica que entre 7 y 8 firmas de auditoría son las que concentran el mercado de los servicios de auditoría en las empresas que cotizan en la bolsa de Brasil; por su parte, en México en recientes años este indicador redunda entre el 0.24 y 0.25 , el cual evidencia que el mercado mexicano de los servicios de auditoría en las empresas que cotizan en la bolsa, está concentrado en 4 firmas.

En Argentina, como ya se citó con antelación, el índice de Concentración que poseen las "Big 4" está entre el 80 y $84 \%$, en tanto que en México este indicador está entre el 87 y $88 \%$.

En Colombia, como ya se mencionó con anteriormente, el Índice de Concentración nivel 4 $\left(\mathrm{IC}_{4}\right)$, está entre el $96 \%$ y el $99 \%$ para recientes años, que es superior al citado indicador mexicano que oscila entre los mencionados 87 y $88 \%$.

\section{CONCLUSIONES}

Con los resultados obtenidos, producto de esta investigación, y con base en lo comentado en los párrafos anteriores, se deja en manifiesto que actualmente en México la concentración en los Servicios de Auditoría ya no es tan elevada como era hace más de 15 años, y que esta concentración mostrada por la nación mexicana ha sido similar a lo acontecido en España, pero menor a la concentración existente en Chile y Colombia. No obstante lo anterior, aún queda una gran área de oportunidad para las Firmas Mexicanas de Auditoría de tamaño mediano, respecto a las estrategias a realizar para intentar incrementar su cuota de participación en el mercado de los servicios del área de auditoría en las empresas que cotizan en la Bolsa de Valores, con lo cual la concentración que existe México pudiera verse disminuida y podría llegar a ser equiparable a la concentración que existe en Argentina y Brasil o a la aún menor concentración promedio que existe en los países de la Unión Europea.

Como referencia para los futuros clientes de estos Despachos, para el momento de la elección de su auditor, importante es recurrir a los hallazgos obtenidos producto de la presente investigación, respecto a los 3 sectores que más domina cada firma:

- DELOITTE: Sector Industrial (subsectores: construcción, bienes de equipo, transportes, suministros y servicios comerciales); sector Materiales (subsector materiales, en los ramos: 
de la construcción, metales y minería, productos químicos, madereros y papeleros); y sector Servicios Financieros (subsector entidades financieras, en los ramos: bancos, grupos financieros, mercados financieros, servicios financieros diversificados $\mathrm{e}$ intermediarios financieros no bancarios).

- KPMG: Sector Servicios Financieros (subsector entidades financieras, en los ramos: bancos, grupos financieros, mercados financieros, servicios financieros diversificados, intermediarios financieros no bancarios y seguros); sector Industrial (subsectores: transportes, construcción y bienes de equipo); y sector Servicios de Telecomunicaciones (subsector medios de comunicación, en el apartado de servicios de radio y televisión).

- EY: Sector Productos de Consumo Frecuente (subsectores: alimentos, bebidas y tabaco y venta de productos de consumo frecuente); sector Servicios Financieros (subsector entidades financieras, en los ramos: grupos financieros, mercados financieros, servicios financieros diversificados y seguros); y sector Materiales (subsector materiales, en los ramos: de la construcción, metales y minería, productos madereros y papeleros).

- PWC: Sector Industrial (subsectores: construcción, transportes, y bienes de equipo); sector Servicios Financieros (subsector entidades financieras, en los ramos: bancos, grupos financieros e intermediarios financieros no bancarios); y sector Servicios y Bienes de Consumo no Básico (subsectores: servicios al consumidor y venta al por menor).

Para futuras investigaciones se podría recomendar el analizar las estrategias que llevan a cabo las Firmas, que no forman parte de las multicitadas "Big 4", que han llegado a auditar a las empresas que cotizan en la BMV, lo anterior para revisar los factores o actividades que han impulsado al éxito o crecimiento de estos Despachos, para que otros puedan tomarlo como referencia y puedan verse beneficiados con esta clase de acciones y con este tipo de investigaciones. 


\section{REFERENCIAS}

Beck, G. (2016). Firma de Auditoría Financiera, Consultoría de Control Interno, Asesoramiento Contable y Tributario. Chile. Recuperado de: http://repositorio.uchile.cl/handle/2250/143339

BDO (2017). Las Mayores Compañías de Auditoría en Argentina. Argentina. Recuperado de: https://www.bdoargentina.com/es-ar/novedades/2017/las-mayores-companias-de-auditoria-en-argentina CFF (2014). Código Fiscal de la Federación. Diario Oficial de la Federación. México. Disponible en: http://dof.gob.mx/nota_detalle.php?codigo=5324942\&fecha=09/12/2013

Cuesta, T., Polo, F. (2005). El mercado de auditoría en España. Análisis de su estructura y evolución. España. https://riunet.upv.es/bitstream/handle/10251/47156/tfc.pdf?sequence=1

Feres, J., Goes, J. (2009). Auditoria e earnings management: estudo empírico nas empresas abertas auditadas pelas big four $e$ demais firmas de auditoria. Brasil. Recuperado de: https://www.scielo.br/j/rcf/a/LvJc7HCvrkQ5HKJgfgCwBsf/?lang=pt

Francis, J., Michas, P., Seavey, S. (2013). Does Audit Market Concentration Harm the Quality of Audited Earnings? Evidence from Audit Markets in 42 Countries. Estados Unidos. Recuperado de: https://www.uts.edu.au/sites/default/files/AccDG_Francis_Michas_Seavey-CAR\%202013.pdf

García-Benau, M., Ruiz, B., Vico, A. (1998). Análisis de la estructura del mercado de servicios de auditoría en España. VI Premio de investigación Contable "José María Fernández Pirla". ICAC, Ministerio Economía y Hacienda, Madrid.

García-Benau, M., Novejarque, J. (2009). Europa quiere potenciar la competencia en el mercado de Auditoría. España. Recuperado de:

http://nicniif.org/files/u57/articulo_para_descargar_pdf_feb_09_5.pdf

Gericó, M. (2018). Las Big Four, ¿verdugos de los grandes despachos de abogados?. España. https://cincodias.elpais.com/cincodias/2017/12/21/legal/1513854824_215410.html

Gutiérrez, B., Pérez, D. \& Barrera, C. (2019). Indicadores del Grado de Concentración de las Firmas de Auditoría en Colombia. México. Recuperado de: http://congreso.investiga.fca.unam.mx/docs/xxiv/docs/5.11.pdf

Heß, B., Stefani, U. (2012). Audit Market Regulation and Supplier Concentration Around the World: Empirical Evidence. Alemania. Recuperado de:

http://www.uni-konstanz.de/FuF/wiwi/workingpaperseries/WP_Hess-Stefani_33-12.pdf

Hernández, A. (2011). Análisis crítico del control interno fiscal y su incidencia en las contingencias fiscales en la industria manufacturera de Nuevo León (Tesis doctoral). Universidad Autónoma de Nuevo León, Facultad de Contaduría Pública y Administración. Nuevo León, México. Recuperado de: http://eprints.uanl.mx/2469/6/1080211160.pdf

Hernández, A., Martínez, B., Luna, A., Hernández, J. (2018). México. La Auditoría de Estados Financieros para efectos Fiscales como Control Interno para reducir las Contingencias Fiscales de las Empresas. Recuperado de:

https://revistainnovaciones.uanl.mx/index.php/revin/article/view/284/276

Hudson, M., Chavink, S., Mos, B. (2014). Big 4 Audit Firms Play Big Role in Offshore Murk. Brasil. https://www1.folha.uol.com.br/internacional/en/business/2014/11/1542579-big-4-audit-firms-play-bigrole-in-offshore-murk.shtml

LMV. (2021). Ley del Mercado de Valores. Diario Oficial de la Federación. México. Recuperado de: http://www.diputados.gob.mx/LeyesBiblio/pdf/LMV_090119.pdf

Lopo, A. Furtado, C. (2020). Tax Aggressiveness and Big4 Audit Firms. Portugal. Recuperado de: https://papers.ssrn.com/sol3/papers.cfm?abstract_id=3599215

Lopo, A., Mendes, G. (2010). Rodízio das firmas de auditoria e o gerenciamento de resultados no brasil. Brasil. Recuperado de: https://www.redalyc.org/pdf/2352/235217196004.pdf

Lopo, A., Mendes, G. (2010). Audit Firm Rotation and Earnings Management in Brazil. Brasil. https://www.researchgate.net/publication/228135440_Audit_Firm_Rotation_and_Earnings_Management_i n_Brazil

Nieves, M., Gutiérrez, I. \& Carmona, S. (2005). Concentración en el Mercado de Auditoría en España: Análisis Empírico del Período 1990-2000. España. https://www.jstor.org/stable/42784073?seq=1 
Price Waterhouse Cooper (2018). Quiénes son las auditoras del IPSA (Indice de Precio Selectivo de Acciones). Chile. Recuperado de: https://www.pwc.com/cl/es/prensa/prensa/2018/Quienes-son-lasauditoras-del-IPSA-PwC-alcanza-a-EY-y-quedaron-empatadas-en-el-primer-lugar.html

Rhode, J., Whitsell, G. \& Kelsey, R. (1974). An Analysis of Client-Industry Concentrations for Large Public Accounting Firms. Estados Unidos. https://www.jstor.org/stable/245055?seq=1

Toscano, J. (2010). Análisis del Mercado de Servicios de Auditoría en México. México. http://www3.uacj.mx/DGDCDC/SP/Documents/RTI/RTI/2.\%20An\%C3\%A1lisis\%20mercado\%20servicio s\%20auditor\%C3\%ADa.pdf

Toscano, J., García, M., Montano, C., Álvarez, C. (2014). El Poder de Mercado y la Concentración Económica en los Servicios de Auditoría Financiera del Mundo. Brasil. https://www.redalyc.org/pdf/1170/117030156009.pdf

Wolk, C., Michelson, S. \& Wootton, Ch. (2001). Auditor Concentration dnd Market Shares in the Us: 1988-1999 A Descriptive Note. Unidos. https://www.sciencedirect.com/science/article/abs/pii/S0890838901901593

Yáñez, R., Ávila, H. (2015). Dictamen de Estados Financieros para Efectos Fiscales: Naturaleza Jurídica, Evolución $\quad e \quad$ Incongruencias. México.

https://www.sciencedirect.com/science/article/pii/S0186104215300073

Zeff, S. \& Fossum, R. (1967). An Analysis of Large Audit Clients. Estados Unidos. Recuperado de: https://www.jstor.org/stable/243935?seq=1 